EPJ Web of Conferences 73, 06006 (2014)

DOI: $10.1051 /$ epjconf/20147306006

(C) Owned by the authors, published by EDP Sciences, 2014

\title{
Ideas of four-fermion operators in electromagnetic form factor calculations
}

\author{
Chueng-Ryong Ji ${ }^{1, \mathrm{a}}$, Bernard L.G. Bakker ${ }^{2, \mathrm{~b}}$, Ho-Meoyng Choi ${ }^{3, \mathrm{c}}$ and Alfredo T. Suzuki ${ }^{4, \mathrm{~d}}$ \\ ${ }^{1}$ Department of Physics, North Carolina State University, Raleigh, NC 27695-8202, USA \\ ${ }^{2}$ Department of Physics and Astrophysics, Vrije Universiteit, De Boelelaan 1081, NL-1081 HV \\ Amsterdam, The Netherlands \\ ${ }^{3}$ Department of Physics, Teachers College, Kyungpook National University, Daegu 702-701, Korea \\ ${ }^{4}$ Instituto de Física Teórica-UNESP Universidade Estadual Paulista, Rua Dr. Bento Teobaldo Ferraz, \\ 271 - Bloco II, 01140-070 São Paulo, SP, Brazil
}

\begin{abstract}
Four-fermion operators have been utilized in the past to link the quark-exchange processes in the interaction of hadrons with the effective meson-exchange amplitudes. In this presentation, we apply the similar idea of Fierz rearrangement to the electromagnetic processes and focus on the electromagnetic form factors of nucleon and electron. We explain the motivation of using four-fermion operators and discuss the advantage of this method in computing electromagnetic processes.
\end{abstract}

\section{Introduction}

Although the calculation of the nucleon form factors based on a quark-diquark model certainly differs from the calculation of the electron form factors using quantum electrodynamics (QED), one may still discern commonalities between the two apparently different calculations. For example, both calculations in the one-loop level share essentially the same shape of triangle diagram as shown in Fig. 1 for the computation of amplitudes. While the contents of the lines drawn in the two triangle diagrams are certainly different, both calculations share the same type of one-loop integration for the amplitudes given by three vertices connected by three propagators. In particular, the structure of the two fermion lines intermediated by a boson exchange is common in the two triangle diagrams and may be generically identified as the four-fermion operator (FFO) that we discuss in this presentation. Due to the commonality of the FFO, it may be conceivable to compute the two apparently different triangle amplitudes in a unified way. As we have recently presented [1], such a unified way of computation is possible since the FFO can be Fierz-rearranged.

\footnotetext{
ae-mail: ji@ncsu.edu

be-mail: b.l.g.bakker@few.vu

ce-mail: homyoung@knu.ac.kr

de-mail: alfredotakashisuzuki@gmail.com
}

This is an Open Access article distributed under the terms of the Creative Commons Attribution License 4.0, which permits unrestricted use, distribution, and reproduction in any medium, provided the original work is properly cited. 


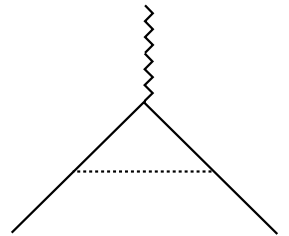

(a)

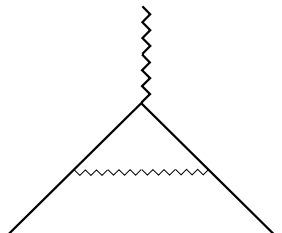

(b)

Figure 1. Triangle diagrams for (a) nucleon form factors in quark-diquark model (b) electron form factors in QED.

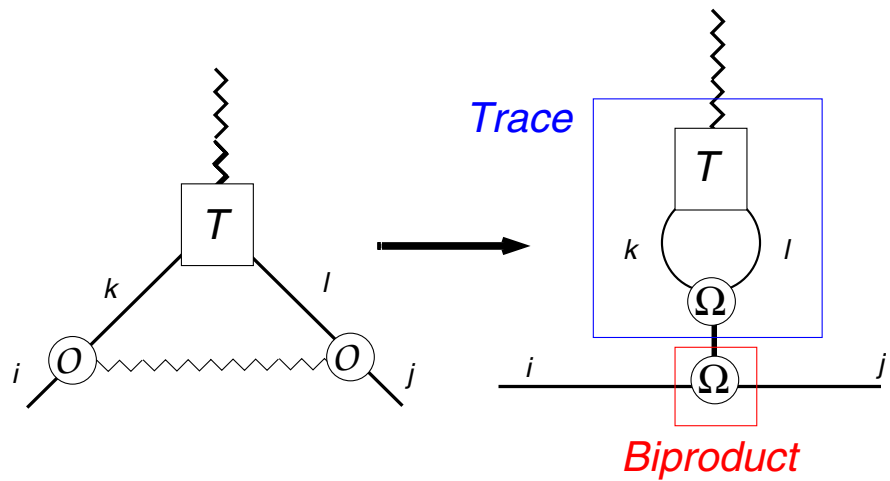

Figure 2. TBasic idea of Fierzrearranged four-fermion operator in electromagnetic processes.

A similar idea of Fierz-rearranged FFOs has been developed in a rather different context of applications in early 1980s. The basic idea of early developments was to provide a basis of the one-boson-exchange interactions of baryons at low energy from the gluon exchange which mediates quark exchange scattering in conjunction with quark interchange in a non-perturbative bag model framework [2-6]. In this presentation, we apply the Fierz-rearranged FFO in the form factors shown in Fig. 1 and present a global formula to cover most (if not all) triangle diagrams for the form factor calculations.

In the next section, Sect. 2, we present the basic idea and a simple illustration of an application using self-energy calculations. In Sect. 3, we apply it to the form factor calculations involving triangle diagrams and present a corresponding global formula. Conclusion and outlook follow in Sect. 4.

\section{Basic idea}

The basic idea of our FFO in electromagnetic processes is depicted in Fig. 2, where an electromagnetic process for a target nucleon is drawn as an illustration. The left and right portions of Fig. 2 correspond to the amplitude intended for computation and the equivalent amplitude after the FFO is Fierz-rearranged, respectively. In the left portion, a single photon is attached to the hadronic part which has the two intermediated quarks denoted by the spinor indices $k$ and $\ell$ that inherit the fermion number from the external nucleons, $\bar{u}_{i}$ and $u_{j}$, with the corresponding spinor indices $i$ and $j$, respectively. Here, the two vertices, $O_{i k}$ and $O_{\ell j}$, connecting the nucleon and the corresponding quark are linked to the scattering amplitude $T_{k \ell}$ where the two photons interact with the constituents from the target nucleon: the rest of the constituents beside the quark is denoted by a wiggly line below the corresponding quark and the loop integration over the internal momentum is understood. From this configuration of the integrand in the amplitude, we may identify the FFO as the multiplication of two vertices $O_{i k} O_{\ell j}$ and rearrange it as

$$
\begin{aligned}
O_{i k} O_{\ell j} & =C_{S} \delta_{i j} \delta_{\ell k}+C_{P}\left(\gamma_{5}\right)_{i j}\left(\gamma_{5}\right)_{\ell k}+C_{V}\left(\gamma_{\mu}\right)_{i j}\left(\gamma^{\mu}\right)_{\ell k}+C_{A}\left(\gamma_{\mu} \gamma_{5}\right)_{i j}\left(\gamma^{\mu} \gamma_{5}\right)_{\ell k}+C_{T}\left(\sigma_{\mu \nu}\right)_{i j}\left(\sigma^{\mu \nu}\right)_{\ell k} \\
& =\Sigma_{\alpha} C_{\alpha} \Omega_{i j}^{\alpha} \Omega_{\ell k}^{\alpha},
\end{aligned}
$$


where $\sigma^{\mu \nu}=\frac{i}{2}\left[\gamma^{\mu}, \gamma^{\nu}\right]$ and the Fierz coefficients $C_{\alpha}(\alpha=S, P, V, A, T)$ depend on the nature of the vertices $O_{i k}$ and $O_{\ell j}$. With this Fierz-rearrangement, we may write the integrand of the amplitude as follows:

$$
\bar{u}_{i} O_{i k} T_{k \ell} O_{\ell j} u_{j}=\Sigma_{\alpha} C_{\alpha}\left(\bar{u}_{i} \Omega_{i j}^{\alpha} u_{j}\right)\left(\Omega_{\ell k}^{\alpha} T_{k \ell}\right)=\Sigma_{\alpha}\left(\bar{u} \Omega^{\alpha} u\right) C_{\alpha} \operatorname{Tr}\left[\Omega^{\alpha} T\right]
$$

where the external nucleon current (or biproduct $\bar{u} \Omega^{\alpha} u$ ) part is now factorized from the internal scattering part given by the trace of the quark loop $\left(\operatorname{Tr}\left[\Omega^{\alpha} T\right]\right)$ as depicted in the right portion of Fig. 2 . With this rearrangement of the same amplitude, one may get the general structure of the target hadron's current more immediately and factorize the details of the internal probing mechanism just due to the relevant constituents for the current of the target hadron. It provides an efficient and unified way to analyze the general structure of the amplitudes sharing the commonality of the same type of diagram for the process.

\section{Application to form factors}

We now apply the idea of FFO and Fierz rearrangement for the form factors shown in Fig. 1 and present the result that covers both the nucleon form factors in a quark-diquark model and the electron form factors in QED. Using the four-fermion method illustrated in Sect. 2 and the usual Feynman parametrization for the loop integration, the current operator $J_{\mu}$ in the amplitude $\bar{u}\left(p^{\prime}\right) J_{\mu} u(p)$ from the triangle diagram with the external fermion mass $M$, the internal fermion mass $m$ and the intermediate boson mass $m_{X}$ can be given by (modulo normalization)

$$
J_{\mu}=\int_{0}^{1} d x \int_{0}^{1-x} d y \int d^{4} k \frac{N_{\mu}}{\left(k^{2}-\Delta^{2}\right)^{3}},
$$

where $\Delta^{2}=s_{+} m^{2}+\left(1-s_{+}\right) m_{X}^{2}-x y q^{2}-s_{+}\left(1-s_{+}\right) M^{2}$ and

$$
\begin{aligned}
N_{\mu}= & C_{S} m\left(1-s_{+}\right)\left(p+p^{\prime}\right)_{\mu}+C_{V}\left[2 k_{\mu} \not k+\frac{\left(1-s_{+}\right)^{2}}{2}\left(p+p^{\prime}\right)_{\mu}\left(p+p^{\prime}\right)\right. \\
& \left.\left.+\left\{m^{2}-k^{2}-\left(1-s_{+}\right)^{2} M^{2}+\left(1-s_{+}+2 x y\right) \frac{q^{2}}{2}\right\} \gamma_{\mu}-\frac{1-s_{-}^{2}}{2} q_{\mu} \phi\right]\right] \\
& +i C_{A} \epsilon_{\mu \nu \alpha \beta} \gamma_{5} \gamma^{\nu}\left(1-s_{+}\right) p^{\alpha} p^{\prime \beta}+2 i C_{T} m \sigma_{\mu \nu} q^{\nu}
\end{aligned}
$$

with $q=p^{\prime}-p$ and $s_{ \pm}=x \pm y$. Although one expects to get $J_{\mu}=\gamma^{\mu} F_{1}\left(q^{2}\right)+i \frac{\sigma_{\mu \nu} q^{v}}{2 M} F_{2}\left(q^{2}\right)$, our result of $J_{\mu}$ appears to exhibit not only the vector and tensor currents but also the scalar and axial vector currents. This can be resolved by the Gordon decomposition and the similar extension such as $\left(p+p^{\prime}\right)_{\mu} \rightarrow 2 M \gamma_{\mu}-i \sigma_{\mu} \nu q^{\nu}$ and $2 i \epsilon_{\mu \nu \alpha \beta} \gamma_{5} \gamma^{\nu} p^{\alpha} p^{\prime \beta} \rightarrow q^{2} \gamma_{\mu}-2 i M \sigma_{\mu \nu} q^{\nu}$, and we get the expected decomposition of $J_{\mu}$ in terms of just vector and tensor currents and find the form factors $(i=1,2)$

$$
F_{i}\left(q^{2}\right)=\int_{0}^{1} d x \int_{0}^{1-x} d y \int d^{4} k \frac{\mathcal{N}_{i}}{\left(k^{2}-\Delta^{2}\right)^{3}},
$$

where

$$
\begin{aligned}
\mathcal{N}_{1}= & 2 M m\left(1-s_{+}\right) C_{S}+\left(1-s_{+}\right) \frac{q^{2}}{2} C_{A} \\
& +\left\{m^{2}+\left(1-s_{+}\right)^{2} M^{2}+\frac{1}{2}\left(1-s_{+}+2 x y\right) q^{2}-\frac{1}{2} k^{2}\right\} C_{V}, \\
\mathcal{N}_{2}= & 4 M m C_{T}-2 M m\left(1-s_{+}\right) C_{S}-2\left(1-s_{+}\right)^{2} M^{2} C_{V}-2\left(1-s_{+}\right) M^{2} C_{A} .
\end{aligned}
$$


The results in Eq. (5) can cover both the nucleon form factors in a quark-diquark model and the electron form factors in QED taking the corresponding Fierz coefficients and masses. For example, $C_{S}=1 / 4, C_{V}=1 / 4, C_{A}=-1 / 4, C_{T}=1 / 8$ if the scalar diquark is taken in the nucleon form factors, while $C_{S}=1, C_{V}=-1 / 2, C_{A}=1 / 2, C_{T}=0$ for the electron form factors in QED. Also, $M, m$ and $m_{X}$ are the nucleon, quark and diquark masses for the nucleon form factors, while $M=m$ is the electron mass and $m_{X}=0$ is the intermediate photon mass in QED calculation of the electron form factors. It is interesting to note that the Fierz coefficient $C_{P}$ neither appear in nucleon form factors nor in electron form factors reflecting the parity conservation both in strong and electromagnetic interactions. In addition, we note that the usual decomposition of $J_{\mu}$ in terms of vector and tensor currents with the Dirac $\left(F_{1}\right)$ and Pauli $\left(F_{2}\right)$ form factors for the nucleon is just one of the six possible decompositions:

$$
\begin{aligned}
J^{\mu} & =\gamma^{\mu} F_{1}+i \frac{\sigma^{\mu \nu} q_{v}}{2 M} F_{2} \\
& =\gamma^{\mu}\left(F_{1}+F_{2}\right)+\frac{\left(p+p^{\prime}\right)^{\mu}}{2 M} F_{2} \\
& =\frac{\left(p+p^{\prime}\right)^{\mu}}{2 M} \frac{4 M^{2} F_{1}+q^{2} F_{2}}{4 M^{2}-q^{2}}-i \epsilon^{\mu \nu \alpha \beta} \gamma_{5} \gamma_{\nu} p_{\alpha} p_{\beta}^{\prime} \frac{2\left(F_{1}+F_{2}\right)}{4 M^{2}-q^{2}} \\
& =\frac{\left(p+p^{\prime}\right)^{\mu}}{2 M} F_{1}+i \frac{\sigma^{\mu \nu} q_{\nu}}{2 M}\left(F_{1}+F_{2}\right) \\
& =\gamma^{\mu}\left(F_{1}+\frac{q^{2}}{4 M^{2}} F_{2}\right)-i \epsilon^{\mu \nu \alpha \beta} \gamma_{5} \gamma_{\nu} p_{\alpha} p_{\beta}^{\prime} \frac{F_{2}}{2 M^{2}} \\
& =i \frac{\sigma^{\mu \nu} q_{\nu}}{2 M}\left(\frac{4 M^{2}}{q^{2}} F_{1}+F_{2}\right)+i \epsilon^{\mu \nu \alpha \beta} \gamma_{5} \gamma_{\nu} p_{\alpha} p_{\beta}^{\prime} \frac{2 F_{1}}{q^{2}} .
\end{aligned}
$$

For the nucleon target, these six different decompositions in Eq. (7) are all equivalent. More details of our form factor analysis using the four-fermion rearrangement idea can be found in our recent paper [1].

\section{Conclusion and outlook}

The idea of rearranging FFOs provides an effective way to analyze hadronic processes. It factorizes the details of the internal probing mechanism from the external global structure due to the target hadrons. Processes sharing a certain commonality (e.g. same type of diagrams) may be described in a unified way as we have shown in the example of electromagnetic form factors. The application to the twophoton processes would be interesting since the generalized hadronic tensor structure of deeply virtual Compton scattering still needs further investigation[7] in view of forthcoming experiments with the $12 \mathrm{GeV}$ upgrade at JLab.

This work is supported by the US Department of Energy (No. DE-FG02- 03ER41260). HMC and ATS acknowledge partial supports from KRF Grant (KRF-2010-0009019) and CNPq (Proc. No. 201.902/2010-9), respectively.

\section{References}

[1] C.-R. Ji, B.L.G. Bakker, H.-M. Choi and A. Suzuki, Phys. Rev. D 87, 093004 (2013)

[2] H.J. Weber, Z. Phys. A 297, 261 (1980); Ann. Phys. (N.Y.) 177, 38 (1987) 
[3] H.J. Weber and J.N. Maslow, Z. Phys. A 297, 271 (1980)

[4] B.L.G. Bakker, M. Bozoian, J.N. Maslow and H.J. Weber, Phys. Rev. C 25, 1134 (1982)

[5] M. Bozoian and H.J. Weber, Phys. Rev. C 28, 811 (1983)

[6] M. Beyer and H.J. Weber, Phys. Lett. B 146, 383 (1984)

[7] C.-R. Ji and B.L.G. Bakker, Int. J. Mod. Phys. E 22, 1330002 (2013) 